\title{
Impact of soil vertical water movement on the energy balance of different land surfaces
}

\author{
Zhiqiu Gao • George Tai-Jen Chen • Yanbing Hu
}

Received: 15 July 2006 /Revised: 26 December 2006 / Accepted: 4 March 2007 / Published online: 12 April 2007

(C) ISB 2007

\begin{abstract}
The soil heat flux determination method proposed by Gao (Boundary-Layer Meteorol 114:165-178, 2005) is discussed for (1) dry surfaces, (2) bare soil or sparse shortgrass lands, and (3) dense-grass surfaces or forest. Our analysis shows that, when neglecting the contribution of soil vertical water movement to soil heat flux, the energy components measured independently will (1) still achieve balance over dry surfaces, and (2) be significantly in imbalance over bare soil or sparse short-grass lands. The mean of bare ground evaporation modeled by $\mathrm{SiB} 2$ is $1.58 \times$ $10^{-5} \mathrm{~m}^{3} \mathrm{~s}^{-1} \mathrm{~m}^{-2}$, and the mean of soil water flux obtained by the method of Gao is $1.22 \times 10^{-5} \mathrm{~m}^{3} \mathrm{~s}^{-1} \mathrm{~m}^{-2}$ for the Naqu site in the summer of 1998. Comparison of the bare ground evaporation with the mean of soil water flux shows a difference, the causes of which are investigated. Physically, the bare ground evaporation is equal to the sum of soil water flux and water content change in the soil surface layer. Because the bare ground evaporation is very limited for the dense-grass surfaces or forest, our analysis implies that the energy imbalance encountered over the dense-grass or forest is not caused by the fact that previous researchers neglected soil water movements in their energy budget analyses.
\end{abstract}

Z. Gao $\cdot$ Y. Hu

State Key Laboratory of Atmospheric

Boundary Layer Physics and Atmospheric Chemistry,

Institute of Atmospheric Physics, CAS,

Beijing, China

Z. Gao $(\bowtie)$

Chinese Academy of Meteorological Sciences,

Box 9804, Beijing 100029, China

e-mail: zgao@mail.iap.ac.cn

G. T.-J. Chen

Department of Atmospheric Sciences,

National Taiwan University,

Taipei, Taiwan
Keywords Land surface energy balance · Soil heat flux . Dry surface $\cdot$ Thermal convection $\cdot$ Soil water movement

\section{Introduction}

Micrometeorologists usually examine the land surface energy balance at a tower flux site, using the ratio of residual $\operatorname{Re}\left(\equiv R n-H-L E-G_{0}\right)$ to available energy (i.e., the difference between the net radiation and soil-canopy surface heat flux, $R n-G_{0}$ ) (e.g., Liu and Foken 2001), where $R n, H$, $L E$, and $G_{0}$ are the net radiation, sensible heat, latent heat and soil heat fluxes, respectively. Table 1 shows some of the results given in previous literature. It is found that (1) the surface energy balance effectively achieves closure over dry surfaces; (2) the surface energy imbalance is significant for bare soil or short-grass land surfaces, and the values of $R e /$ $\left(R n-G_{0}\right)$ are higher than $30 \%$; and (3) a surface energy imbalance is also encountered over vegetated soils, mediumgrass lands or forest, and the values of $\operatorname{Re}\left(R n-G_{0}\right)$ ranged from $10 \%$ to $20 \%$.

Soil heat flux is an important component of the land surface energy balance (e.g., Kustas et al. 1999, 2000). Gao (2005) proposed a method to calculate soil heat. This method differs from traditional methods because it accounts for both soil thermal conduction and soil thermal convection. Using the data collected during the Global Energy and Water cycle Experiment (GEWEX), Asian Monsoon Experiment (GAME)-Tibet intensive observation period from mid-July to mid-September in 1998, Gao (2005) compared the energy balance flux components and energy budget closure calculated by the traditional methods with those by his methods, and found that the energy budget closure $\varepsilon$ (defined as the ratio of the sum of sensible and latent heat fluxes to available energy) was 0.7 and 0.9 for the traditional and his method, 
Table 1 Comparison of the land surface energy imbalance $\Delta\left(\equiv \operatorname{Re} /\left(R n-G_{0}\right) \times 100\right)$ or the residuum energy $(\mathrm{Re})$, obtained from different land-cover classes

\begin{tabular}{|c|c|c|}
\hline Experiment and/or reference & $\Delta$ or $\operatorname{Re}$ & Underlying surface \\
\hline \multicolumn{3}{|l|}{ Dry surfaces } \\
\hline Albertson et al. (1995) & 0 & Dry Owens Lake bed \\
\hline Unland et al. (1996) & 0 & Semi-arid Sonoran Desert \\
\hline Heusinkvld et al. 2004 & 0 & Sandy desert \\
\hline \multicolumn{3}{|l|}{ Bare soils or short-grass land surfaces } \\
\hline TARTEX-90 (Foken et al. 1993) & 33 & Barley and bare soil \\
\hline KUREX-91 (Panin et al. 1996) & 33 & Different surfaces \\
\hline LINEX-97/1 (Foken 1998) & 32 & Short grass \\
\hline LITFASS-98 (Beyrich et al. 2002) & 37 & Nearly bare soil \\
\hline GAME/Tibet-98 (Gao 2005) & 30 & Sparse short grass \\
\hline \multicolumn{3}{|l|}{ Vegetated soils, medium-grass lands or forest } \\
\hline FIFE-89 (Kanemasu et al. 1992) & 10 & Steppe, grassland \\
\hline Vancouver I-90 (Lee and Black 1993) & 17 & $16-\mathrm{m}$ forest \\
\hline LINEX-96/2 (Foken et al. 1997) & 20 & Medium grass \\
\hline FLUXNET (Wilson et al. 2002) & 20 & $\begin{array}{l}\text { Tundra, conifer, agriculture } \\
\text { forest, grassland et al. }\end{array}$ \\
\hline Kelliher et al. 1992 & $\operatorname{Re}=50-150 \mathrm{Wm}^{-2}$ & Broad-leaved forest \\
\hline Oncley et al. 2002 & $\begin{array}{l}\mathrm{Re}=110 \mathrm{Wm}^{-2}=16 \% \\
\text { of } \mathrm{Rn}\end{array}$ & Forest \\
\hline
\end{tabular}

respectively. It is important to note that Gao's method was originally designed for land surfaces where the soil vertical water movements is significant.

In order to provide guidance to the modeling community on how to interpret flux measurements that do not appear to be consistent with the conservation principles (Kustas et al. 1999), the micrometeorological measurement community has been attempting to resolve these serious discrepancies in the surface energy balance closure (Twine et al. 2000). However, few previous articles have considered the phenomenon that the surface energy balance achieves closure well over dry hot surfaces while energy imbalance is encountered frequently for other types of land surface. The present research builds on the work of Gao (2005), and attempts to explain why energy balance achieves closure well over a dry surface. The second goal of this paper is to better understand the energy balance for different land surfaces by comparing soil water flux to bare ground evaporation rate using the data utilized in Gao (2005).

\section{Materials and methods}

Review of Gao et al. (2003) and Gao (2005)

\section{Soil temperature}

Gao et al. (2003) incorporated thermal conduction and convection equations to consider these two processes together as follows,

$\frac{\partial T}{\partial t}=k \frac{\partial^{2} T}{\partial z^{2}}+W \frac{\partial T}{\partial z}$, where $k$ is the soil thermal diffusivity and $W$ is the liquid water flux density. Given the boundary condition: $\left.\mathrm{T}\right|_{\mathrm{z}=0}=\overline{\mathrm{T}}+\mathrm{A} \sin \omega \mathrm{t},(\mathrm{t} \geq 0)$, where $\overline{\mathrm{T}}$ is a constant, $A$ is the amplitude of the soil surface temperature, and $\omega$ is the angular velocity of the Earth's rotation, they derived the solution to Eq. (2) as follows,

$$
\begin{aligned}
T(z, t)= & T_{0}+A \\
& \times \exp \left[\left(-\frac{W}{2 k}-\frac{\sqrt{2}}{4 k} \sqrt{W^{2}+\sqrt{W^{4}+16 k^{2} \omega^{2}}}\right) z\right] \\
& \cdot \sin \left[\omega t-z \frac{\sqrt{2} \omega}{\sqrt{W^{2}+\sqrt{W^{4}+16 k^{2} \omega^{2}}}}\right]
\end{aligned}
$$

Assuming $A_{1}, A_{2}$ are amplitudes, and $\Phi_{1}, \Phi_{2}$ are initial phases of soil temperatures at the depths of $z_{1}$ and $z_{2}$ in the soil, respectively, and assuming $\mathrm{z}_{1}>\mathrm{z}_{2}$ (i.e., $\mathrm{A}_{1}<\mathrm{A}_{2}$ and $\Phi_{1}>\Phi_{2}$ ), Gao (2005) derived the followed equations.

$$
\begin{aligned}
\mathrm{W} & =\frac{\omega\left(z_{1}-z_{2}\right)}{\Phi_{1}-\Phi_{2}}\left[\frac{-\left(\Phi_{1}-\Phi_{2}\right)^{2}+\ln ^{2}\left(\mathrm{~A}_{1} / \mathrm{A}_{2}\right)}{\left(\Phi_{1}-\Phi_{2}\right)^{2}+\ln ^{2}\left(\mathrm{~A}_{1} / \mathrm{A}_{2}\right)}\right] \\
& =\frac{\omega\left(z_{1}-z_{2}\right)}{\Phi_{1}-\Phi_{2}}\left[\frac{2 \ln ^{2}\left(\mathrm{~A}_{1} / \mathrm{A}_{2}\right)}{\left(\Phi_{1}-\Phi_{2}\right)^{2}+\ln ^{2}\left(\mathrm{~A}_{1} / \mathrm{A}_{2}\right)}-1\right],
\end{aligned}
$$

and

$$
k=-\frac{\left(z_{1}-z_{2}\right)^{2} \omega \ln \left(A_{1} / A_{2}\right)}{\left(\Phi_{1}-\Phi_{2}\right)\left[\left(\Phi_{1}-\Phi_{2}\right)^{2}+\ln ^{2}\left(A_{1} / A_{2}\right)\right]} .
$$




\section{Land surface energy budget closure}

Surface soil heat flux $\left(G_{0}\right)$ is usually estimated by adding the heat storage of the soil layer above the plate to the plate measurements, namely

$\mathrm{G}_{0}=\mathrm{G}_{1}+\mathrm{C}_{\mathrm{g}} \Delta z \partial \mathrm{T} / \partial \mathrm{t}$,

where $G_{0}$ is the surface soil heat flux, $G_{1}$ is the soil heat flux at depth (from the plate heat flow measurement in soil), $\mathrm{C}_{\mathrm{g}}$ is the volumetric heat capacity of the soil, which can be easily derived from soil components (Van Wijk 1963), $\Delta \mathrm{z}$ is the thickness of a thin layer of the soil and $T$ the mean soil temperature of the thin layer, $\partial \mathrm{T}$ is the change in mean soil temperature during the measurement period, and $\partial \mathrm{t}$ the change in time. $C_{g} \Delta z \partial T / \partial t$ represents the heat storage of the soil layer.

Applying Eq. (1) to Eq. (5) gives

$$
G_{0}=G_{1}+C_{g} k \partial T / \partial z+C_{w} W \Delta T
$$

where $C_{w}$ is the water volumetric heat capacity. Gao (2005) pointed out that, physically, $C_{g} k \partial T / \partial z$ contributes to the soil heat flux and depends on thermal diffusivity, and $C_{w} W \Delta T$ contributes to the soil heat flux through thermal convection within the surface soil layer. Gao (2005) therefore suggested the surface energy balance equation to be

$R_{n}=H+L E+G_{1}+C_{g} k \partial T / \partial z+C_{w} W \Delta T$ where $R_{n}, \mathrm{H}$ and $\lambda \mathrm{E}$ are net radiation, sensible heat flux, and latent heat flux, respectively, all which can be obtained by direct measurements.

New derivation for dry surfaces

\section{Soil temperature}

We assume $\mathrm{W}=0$ for dry surfaces, such as a dry hot desert, because $W$ decreases with decreasing soil moisture (Gao 2005) and the soil moisture is extremely low there (Heusinkvld et al. 2004). In this way, Eq. (2) becomes

$$
T(z, t)=T_{0}+A \exp (-z \sqrt{\omega / 2 k}) \sin (\omega t-z \sqrt{\omega / 2 k}),
$$

Equation (8) is widely used in textbooks (e.g., Stull 1988; Garratt 1992) for calculation of soil temperature. It means that the amplitude of the soil temperature exponentially decreases and the phase of the soil temperature delays linearly when the soil depth increases. Because Gao (2005) gave an explicit mathematical derivation and a physical explanation to Eq. (2) , we believe that Eq. (2) reasonably describes the variation of soil temperature with soil depth. Comparing Eq. (8) with Eq. (2) means that a model derived from Eq. (8) will (1) generate a reasonable result of soil temperature for a dry hot sandy desert; and (2) overestimate
Fig. 1 a Inter-comparison of the measured $(H+\lambda \mathrm{E})$ against available energy $\left(\mathrm{G}_{1}+\mathrm{G}_{2}\right)$ using previous method, and $\mathbf{b}$ intercomparison of the measured $(H+\lambda \mathrm{E})$ against available energy $R_{n}-G_{1}-G_{3}-G_{4}$ using present method at the Naqu site in the Tibetan Plateau from DOY 195 to DOY 258, 1998. (After Gao 2005)
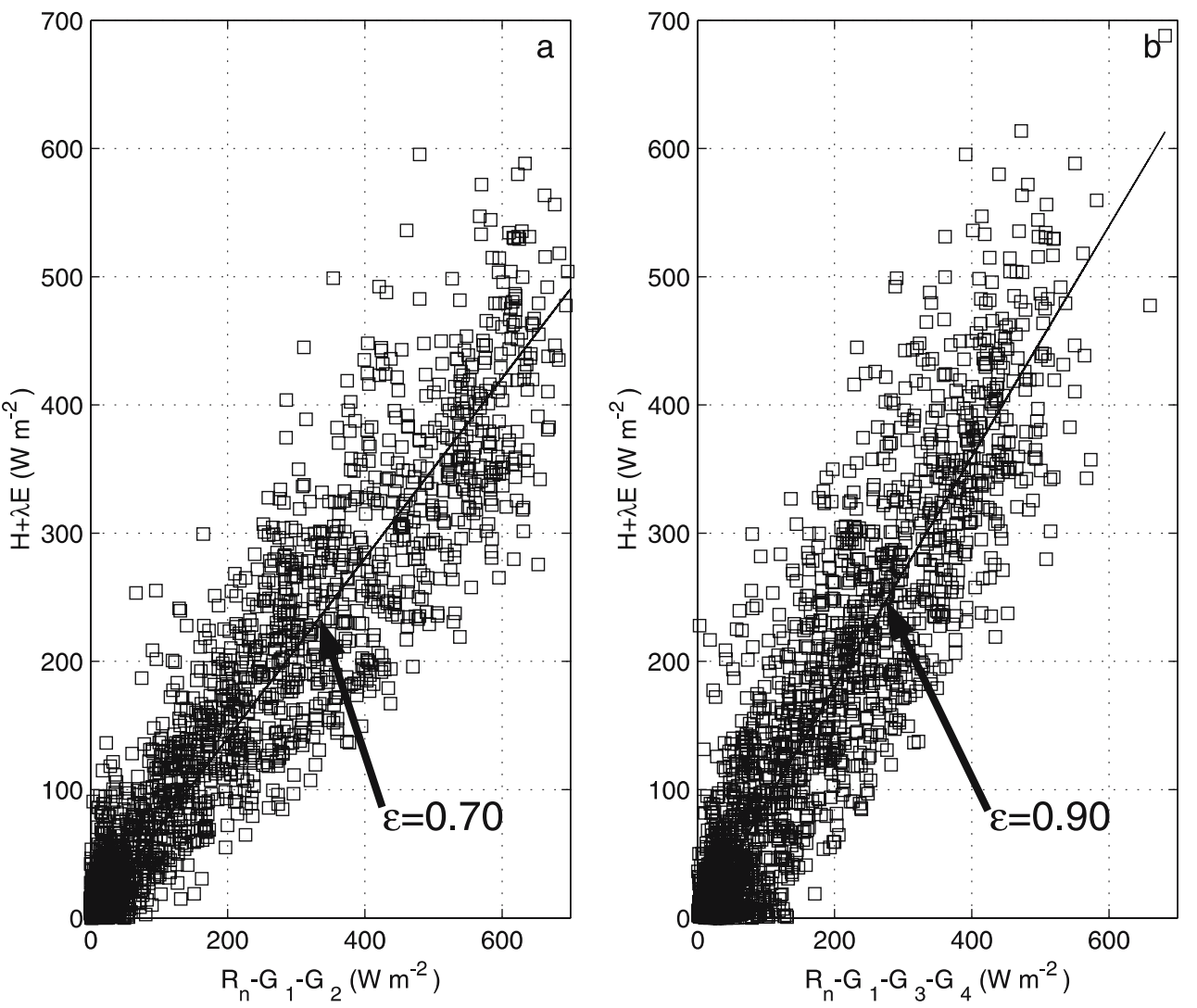
the amplitude of soil temperature, and delay the phase of soil temperature for other surfaces where $W \neq 0$, such as bare soil and short-grass lands.

When $W=0$, Eq. (3) means

$\Phi_{1}-\Phi_{2}=-\ln \left(A_{1} / A_{2}\right)=\ln \left(A_{2} / A_{1}\right)$,

and Eq. (4) therefore becomes

$k=\frac{\left(z_{1}-z_{2}\right)^{2} \omega}{2\left(\Phi_{1}-\Phi_{2}\right)^{2}}=\frac{\left(z_{1}-z_{2}\right)^{2} \omega}{2 \ln ^{2}\left(A_{1} / A_{2}\right)}$.

Because $z_{1} \neq z_{2}$, Eq. (10) means that $\mathrm{k}>0$ always. Eqs. (9-10) imply, for a dry hot desert $(W=0),(1)$ that the phase delay equals to the logarithm of amplitude ratio of soil temperature collected at two depths; (2) that thermal conduction still occurs whereas thermal convection does not happen when $W=0$; and (3) that soil thermal diffusivity $k$ can be determined using amplitudes or phases of soil temperature collected at two depths of dry sandy soil. If surface temperature is used, i.e., $\mathrm{z}_{2}=0, \Phi_{2}=0$, and $A_{2}=A$, Eq. (10) can be simplified to

$k=\frac{z_{1}^{2} \omega}{2 \Phi_{1}^{2}}=\frac{z_{1}^{2} \omega}{2 \ln ^{2}\left(A_{1} / A\right)}$,

which implies that the soil thermal diffusivity $k$ can be determined by using one-level soil temperature when soil surface temperature is derived from the long-wave radiation omitted from the soil surface.

\section{Surface energy balance budget}

The same principle applies to Eq. (6). When $W=0$, Eq. (6) becomes

$G_{0}=G_{1}+C_{g} k \partial T / \partial z$

and Eq. (7) becomes

$R_{n}=H+L E+G_{1}+C_{g} k \partial T / \partial z$,
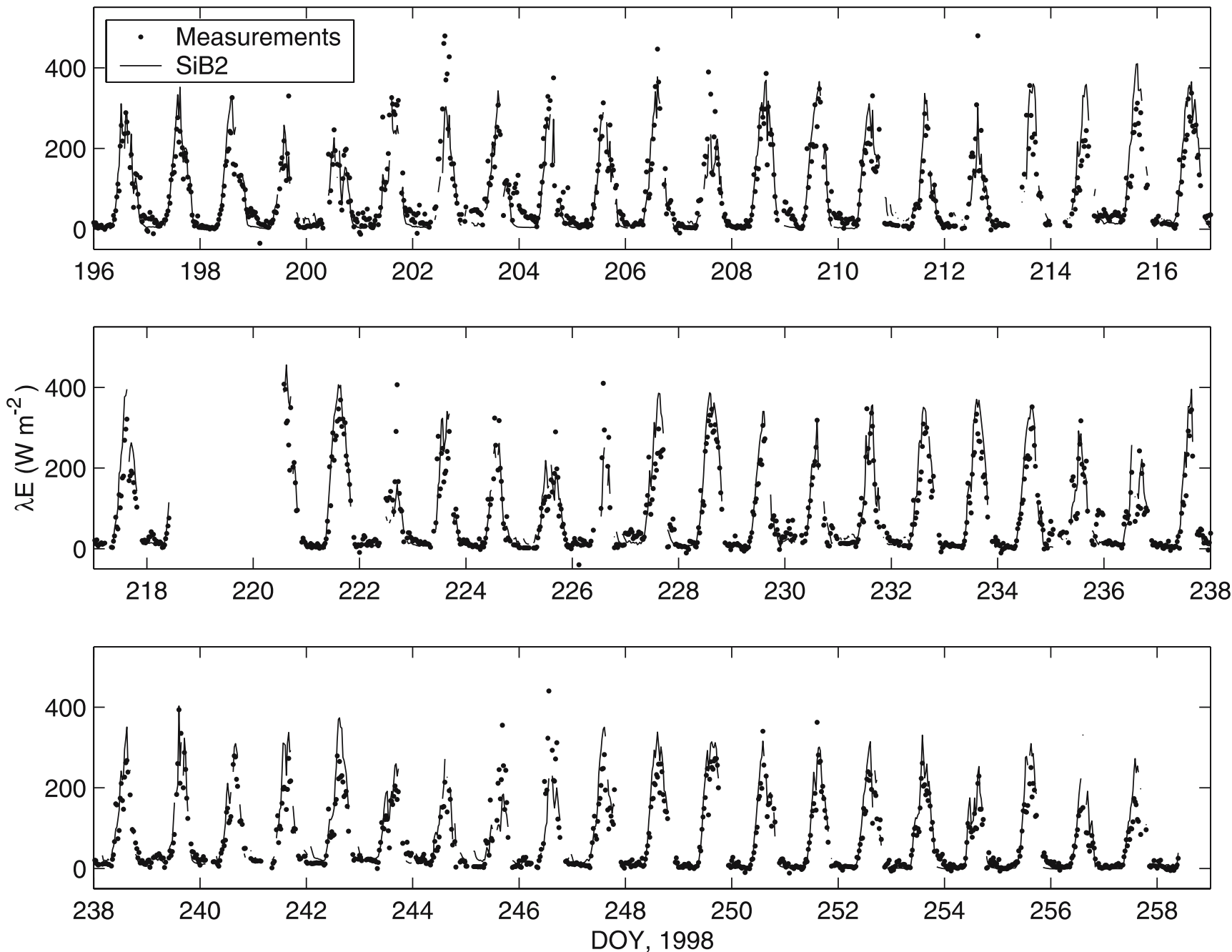

Fig. 2 Comparison of latent heat flux $(\lambda \mathrm{E})$ modeled using SiB2 against direct measurements. (After Gao et al. 2004) 
which is also widely given in textbooks (e.g., Stull 1988) for calculation of soil heat flux. It means that the heat absorbed by the soil surface vertically transfers into the deep soil by thermal conduction only. So comparing Eq. (11) with Eq. (6) means that a model derived from Eq. (11) will (1) generate a reasonable result of soil heat flux, when it is applied to a dry hot sandy desert $(W=0)$; and (i2 underestimate soil heat flux, when it is applied to other surfaces where $W \neq 0$, such as bare soil and shortgrass surfaces.

\section{Results}

Soil temperature for a dry hot desert

Because we have no data collected for dry soils, we have to use the illustration existing in previous literature for our analysis. Heusinkvld et al. (2004) analyzed the surface energy balance for a sandy desert. Because the average annual rainfall was $100 \mathrm{~mm}$ and there were sparsely vegetated linear dunes at their site, the soil moisture did not reach zero, with the result that the soil temperatures modeled by Heusinkvld et al. (2004) had somewhat larger amplitudes, and had somewhat delayed phases for all four depths $(0.015 \mathrm{~m}, 0.034 \mathrm{~m}, 0.05 \mathrm{~m}$ and $0.1 \mathrm{~m})$, as shown in Heusinkvld et al. (2004, Fig. 5).
Energy budget closure over land surfaces

\section{Dry surface}

We have pointed out that a model neglecting vertical soil water movement will generate a reasonable estimation of energy budget closure for a dry hot desert where $W$ is extremely low, so it is not a surprise that energy balance closure was found to be very good at the site of Heusinkvld et al. (2004). Besides this, Albertson et al. (1995) examined the components of energy budget balance over the dry Owens Lake bed in Owens Valley, California, where the underlying surface is crusted sand with substantial amounts of evaporative salts. It is obvious that Albertson et al. (1995) did not account for the vertical soil water movements. However, their results (i.e., their Fig. 2a,b) show, not surprisingly, that energy budget was almost achieved closure at their site. Furthermore, Unland et al. (1996) examined surface flux measurements and model output at a semi-arid Sonoran Desert site, and their results show that the energy balance closure was found to be good, too, as shown in Unland et al. (1996, Fig. 5).

\section{Bare soil or sparse short-grass lands}

Our analysis above implies that if the vertical soil water movement is neglected in the calculation of soil heat flux $\left(G_{0}\right)$,
Fig. 3 Scatter plot of latent heat flux $(\lambda \mathrm{E})$ modeled using $\mathrm{SiB} 2$ against direct measurements

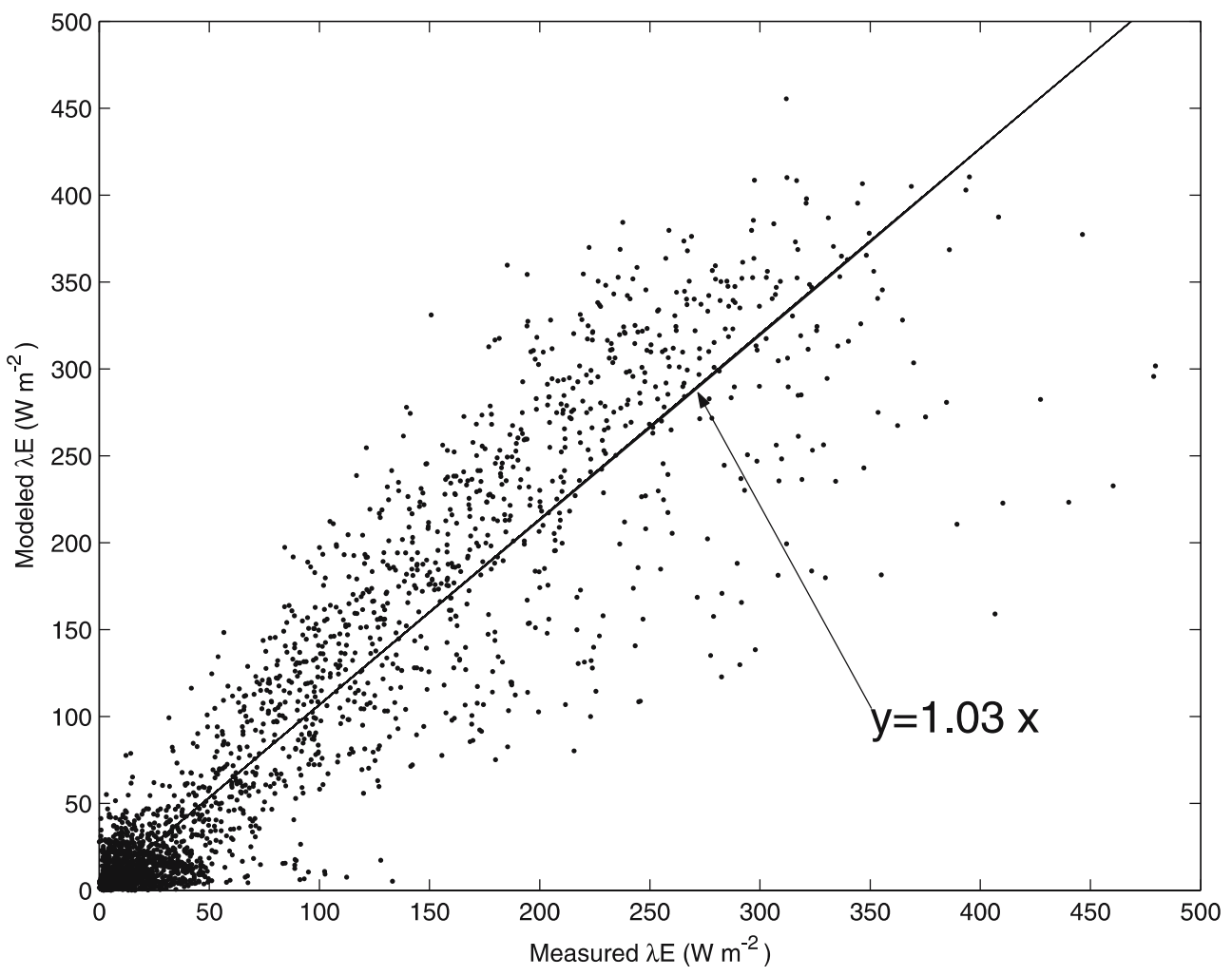


a significant energy imbalance will be encountered for bare soils or short-grass lands where the vertical soil water movement is considerable. Table 1 gives values of $\mathrm{Re} /(\mathrm{Rn}-$ $\left.\mathrm{G}_{0}\right) \cdot 100 \%$ for the bare soil or short-grass land surfaces where the soil vertical water movement was not considered. It is obvious that the energy imbalance is significant and that values of $\mathrm{Re} /\left(\mathrm{Rn}-\mathrm{G}_{0}\right) \cdot 100 \%$ are larger than $30 \%$. Note that the energy imbalance reaches $30 \%$ for a Tibetan short-grass prairie as shown in Table 1. Gao (2005) re-examined the soil heat flux for that site by considering soil vertical water movement, resulting in $\mathrm{Re} /\left(\mathrm{Rn}-\mathrm{G}_{0}\right) \cdot 100 \%$ being only $10 \%$, as shown in Fig. 1.

Gao et al. (2004) simulated surface energy partitioning in the Tibetan prairie using the simple biosphere model 2 ( $\mathrm{SiB} 2$ ), and the modeled values for the 2-month period were compared with micrometeorological field measurements made through the GAME-Tibet IOP 1998. The results show that, when underestimating net radiation by $11 \%$, the model overestimated sensible, latent, and soil heat fluxed by 8,3 , and $13 \%$ respectively. Because we are just concerned about soil water continuity, here we only give the modeled results of latent heat flux as shown in Fig. 2. We found that SiB2 significantly underestimated latent heat flux around 1200 hours on DOYs 202, 204, 207, 212, 222, 226, 245, and 246, although it gave reasonable estimates of latent heat flux for all other times. Figure 3 is the scatter plot of latent heat flux $(\lambda \mathrm{E})$ modeled using $\mathrm{SiB} 2$ against direct measurements. It shows that on average $\lambda \mathrm{E}$ was overestimated by $3 \%$ with the correlation coefficient of 0.92 .

The latent heat flux $(\lambda \mathrm{E})$ modeled by $\mathrm{SiB} 2$ consists of two parts: $\lambda \mathrm{E}_{c}$ and $\lambda \mathrm{E}_{g}$, where $\lambda \mathrm{E}_{c}$ is from the canopy and $\lambda \mathrm{E}_{g}$ is from the bare soil surface. $\lambda$ is the latent heat of vaporization, $E_{c}$ is the evapotranspiration rate from canopy and $E_{g}$ is the evaporation from the bare soil surface. The temporal variations of $\lambda \mathrm{E}_{c}$ and $\lambda \mathrm{E}_{g}$ are shown in Fig. 4 . We found that the magnitude of $\lambda \mathrm{E}_{c}$ is close to that of $\lambda \mathrm{E}_{g}$ although, during the monsoon season, the mean canopy height was just $0.05 \mathrm{~m}$ and the leaf area index (hereinafter,
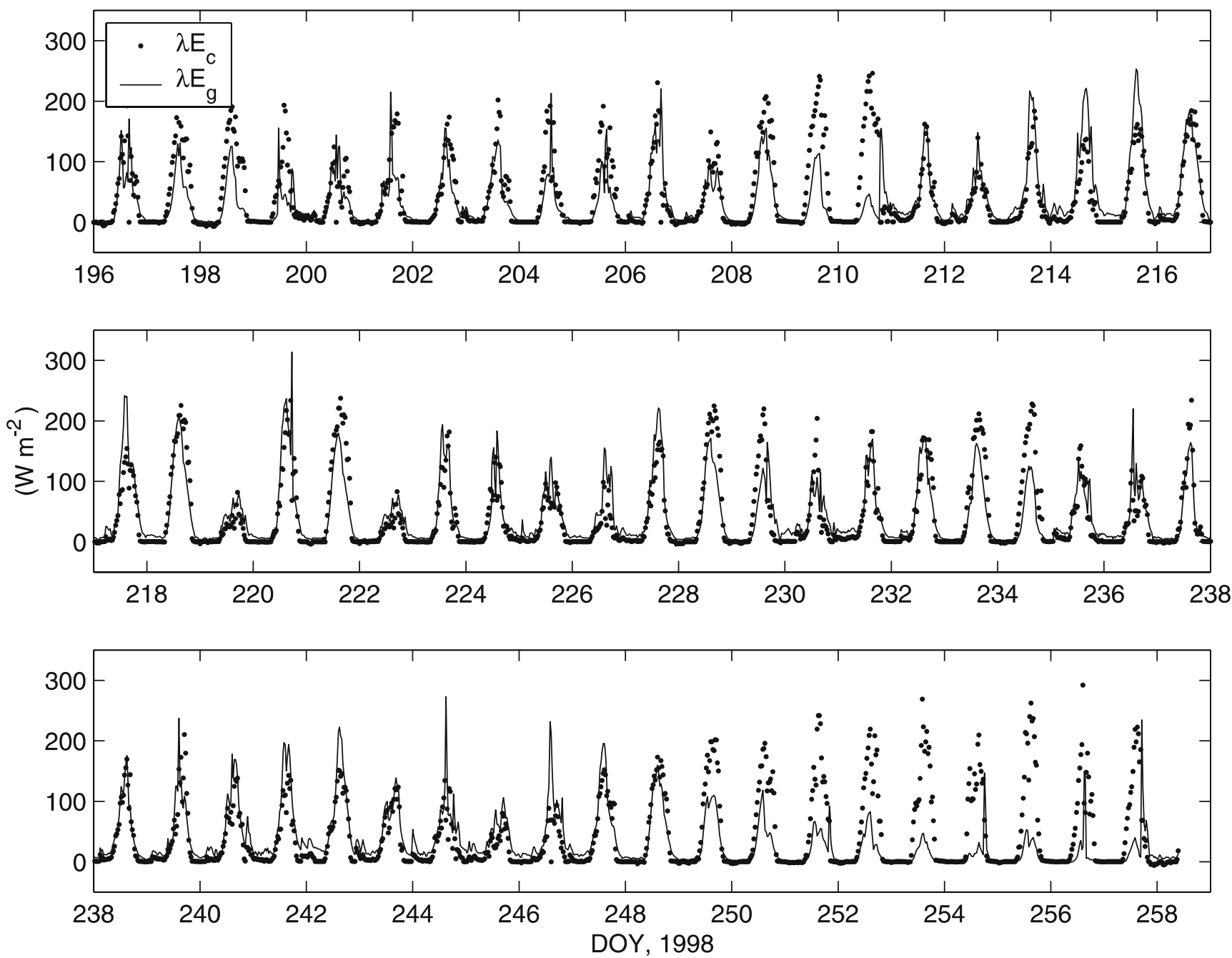

Fig. 4 Temporal variations of latent heat flux from canopy $\left(\lambda \mathrm{E}_{c}\right)$ and latent heat flux from bare ground $\left(\lambda \mathrm{E}_{g}\right)$ at the Naqu site in the Tibetan Plateau from DOY 195 to DOY 258, 1998 
referred to as LAI) were assumed to be 0.5 , respectively. Therefore, we deduced that $\lambda \mathrm{E}_{c}$ would be more significant than $\lambda \mathrm{E}_{g}$ for vegetated surfaces where LAI is much larger.

Because of soil water continuity in the vertical direction, in theory the daily average of the bare surface evaporation $\left(E_{g}\right)$ should be equal to the sum of soil water flux $(W)$ and water content change in the soil surface layer, although the phase change of water happens in the soil surface layer. $W$ is calculated from the temperature measurements at two depths in soil (Gao 2005). Comparison of bare ground evaporation $\left(E_{g}\right)$ against soil water flux $(W)$ is shown in Fig. 5. It was found that soil water flux $(W)$ is less and approaches the case of bare surface evaporation $\left(E_{g}\right)$; on average, $E_{g}=1.58 \times$ $10^{-5} \mathrm{~m}^{3} \mathrm{~s}^{-1} \mathrm{~m}^{-2}$ and $\mathrm{W}=1.22 \times 10^{-5} \mathrm{~m}^{3} \mathrm{~s}^{-1} \mathrm{~m}^{-2}$. The difference between $E_{g}$ and $W$ is caused by the following reasons:

1.) The difference was mainly caused by water content change in the soil surface layer. Because the whole observation period is within the monsoon season, rain events happened frequently (Gao 2005). The cold rainwater infiltrating into the soil created complexities in soil temperature distribution.

2.) Because it was impossible for us to directly measure soil evaporation from the bare ground $\left(E_{g}\right)$, we have to use the modeled $E_{g}$ for our comparison. Uncertainties of modeling bare surface evaporation $\left(E_{g}\right)$ negatively affect the comparison results.
3.) Equation (3) shows that the soil water flux ( $W$ ) depends significantly on the phase difference and on the amplitude ratio of soil temperatures collected at two depths, so accurate determination of the soil temperature phase and the soil temperature amplitude therefore becomes essential. The error in determination of the soil temperature phase and the soil temperature amplitude also negatively affect the comparison results.

\section{Grass-land surfaces or forest}

Over the surfaces which were well vegetated, underlying surface energy absorption happens mainly at the vegetation canopy rather than at the soil surface. Table 1 gives values of $\mathrm{Re} /\left(\mathrm{Rn}-\mathrm{G}_{0}\right) \cdot 100 \%$ for vegetated soils, medium-grass lands and forest where the vertical soil water movement was not considered. It is obvious that values of $\mathrm{Re} /\left(\mathrm{Rn}-\mathrm{G}_{0}\right) \cdot 100 \%$ ranged from $10 \%$ to $20 \%$.

There could be a number of reasons for the energy imbalances listed in the Table 1. Over vegetated surfaces, the canopy evapotranspiration flux contributes a significant part to the total latent heat flux, which means the soil water is withdrawn directly by the root systems. Wilson et al. (2002) gave a comprehensive evaluation of energy balance closure at FLUXNET sites. Their results show a general lack of closure at most sites, with a mean imbalance in the
Fig. 5 Comparison of evaporation from bare ground $\left(E_{g}\right)$ against soil water flux $(W)$ at the Naqu site in the Tibetan Plateau from DOY 195 to DOY 258, 1998

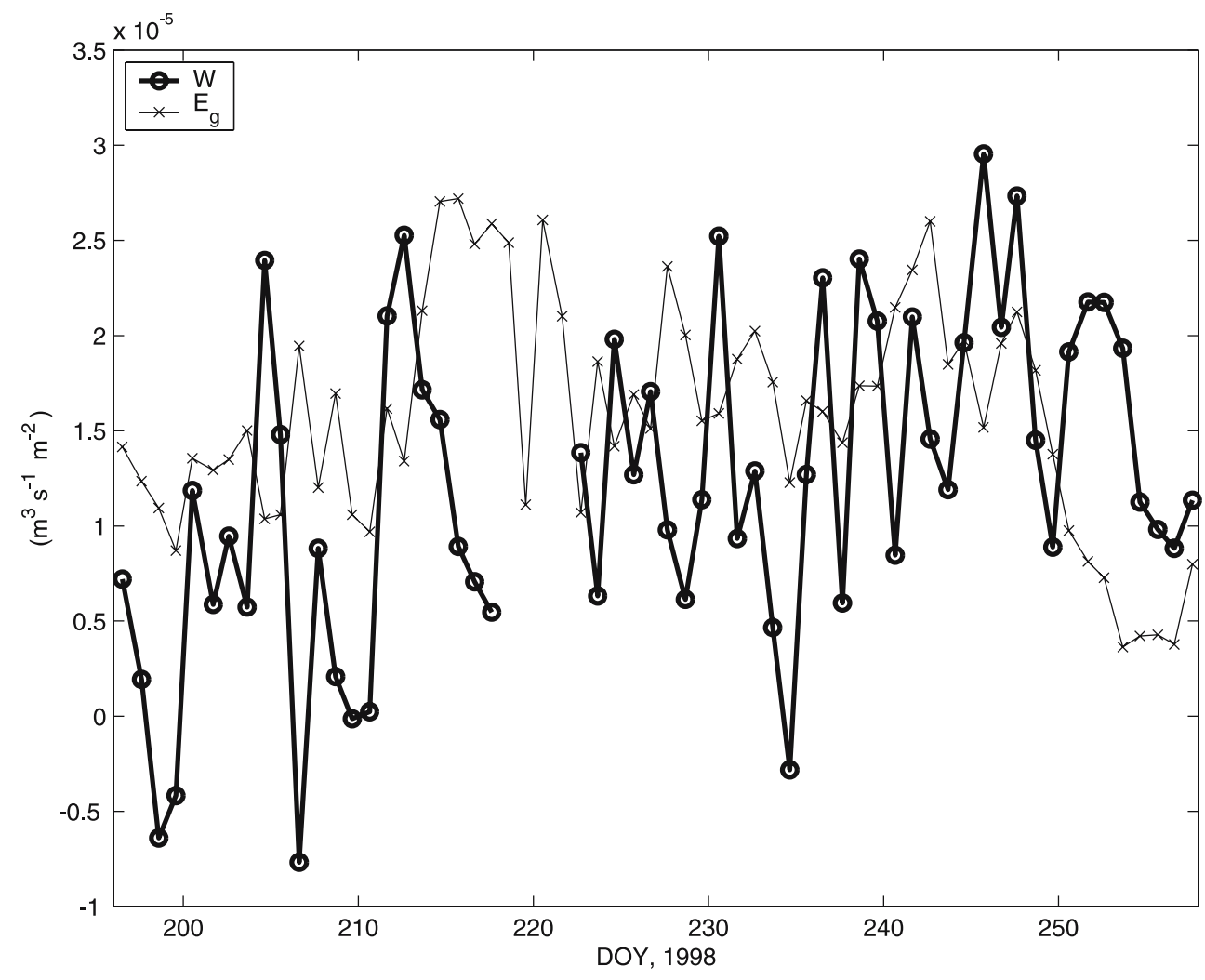


order of $20 \%$, and they concluded that the sum of sensible heat flux and latent heat flux was underestimated and/or that available energy was overestimated. We certainly agree with them. Our above analysis implies that the magnitude of soil water flux $(W)$ is less than and approaches that of bare soil surface evaporation $\left(E_{g}\right)$. The bare soil surface evaporation $\left(E_{g}\right)$ is insignificant over dense-grass surface or forest, so the soil water movement plays a minor role in surface energy balance there.

\section{Discussion and conclusions}

Tanaka et al. (2001, 2003) and Ma et al. (2003) investigated surface energy partitioning over the Tibetan Plateau by using the data collected during the GAME-Tibet IOP 1998. They pointed out that the surface energy imbalance was caused by measurement uncertainty of latent heat flux at their sites. Table 1 also supports their conclusion, because Table 1 shows that the energy imbalance is between $10 \%$ and $20 \%$ over the vegetated soil, medium-grass lands or forest where the latent heat flux is the main consumer of available energy. The present work mainly attempts to explain why the energy imbalance exceeds $30 \%$ of available energy for wet sparse short-grass land or wet bare soil, where latent heat flux is an important energy consumer of surface available energy and the magnitude of the evapotranspiration rate from canopy $\left(E_{c}\right)$ is roughly close to that of, and the evaporation from, the bare soil surface $\left(E_{g}\right)$.

Lee (1998) investigated the surface-air exchange over tall vegetation, and concluded that energy imbalance is caused by the energy advection. We think that the energy advection is important for tall vegetation such as forest, but it is not important for the wet sparse short-grass land or wet bare soil.

This paper explains why the energy balance achieves closure for a dry sandy desert while energy imbalance was encountered for other types of land surface, when traditional methods are used for determination of energy balance components. Furthermore, we classify the land surfaces into three groups: (1) dry and bare soil; (2) wet sparse shortgrass land or wet bare soil; and (3) well vegetated soil, medium-grass lands or forest. Previous work indicates that energy balance is achieved for dry and bare soil surfaces; energy imbalance exceed $30 \%$ of available energy for wet sparse short-grass land or wet bare soil; and the energy imbalance is between 10 and $20 \%$.

The traditional method of calculating soil heat does not account for the influence of soil vertical water movements on soil surface temperature and on soil heat flux. Although it can be used for dry and bare soil, where the vertical water movement is insignificant, it significantly underestimates the soil heat flux for wet sparse short-grass land or bare soil where the vertical water movement is significant.
The direct evaporation from the bare part of the surface is not significant over densely vegetated surfaces, so we conclude that the water movement plays a minor role in the surface energy balance. Work will continue to identify the source of the energy imbalance encountered over densely vegetated surfaces.

Acknowledgements This study was supported by MOST (2006CB400500 and 2006CB403600), by NSFC (40575007 and 40333032), and by the CAS Centurial Program. We would like to acknowledge authors of references published in the last 10 years. Their papers make us aware of the confusion surrounding land surface energy balance. This has allowed us the opportunity to clarify the influence of soil vertical water movements on surface energy balance for different underlying surfaces. Special thanks go to Prof. Kim Joon and his students Dr. Choi Taejin and Lee Heechoon for their dedication and excellent work in data collection. I am very grateful to Dr. Liu Heping for his comments which led to substantial improvements of this manuscript. I thank Professor Wang Jiemin for discussion. We are very grateful to anonymous reviewers for their careful review and valuable comments, which led to substantial improvement of this manuscript.

\section{References}

Albertson JD, Parlange MB, Katul GG, Chu CR, Stricker H (1995) Sensible heat flux from arid: a simple flux-variance method. Water Resour Res 31:969-973

Beyrich F, Foken, T, Herzog HJ (2002) The LITFASS-98 experiment. Theor Appl Climatol 73:1-2

Foken T, Gerstmann W, Richter SH, Wichura B, Baum W, Ross J, Sulev M, Mölder M, Tsvang LR, Zubkovskii SL, Kukharets VP, Aliguseinov AK, Perepelkin VG, Zeleny J (1993) Study of the energy exchange processes over different types of surfaces during TARTEX-90. Deutscher Wetterdienst, Forschung und Entwicklung, Arbeitsergebnisse 4:34. [available from German Weather Service

Foken T, Jegede OO, Weisensee U, Richter SH, Handorf D, Gördorf U, Vogel G, Schubert U, Kirzel HJ, Thiermann V (1997) Results of the LINEX-96/2 Experiment. Deutscher Wetterdienst, Forschung und Entwicklung, Arbeitsergebnisse 48:75. [available from German Weather Service]

Foken T, Buck AL, Nye RA, Horn RD (1998) A Lyman-alpha hygrometer with variable path length. J Atmosph Oceanic Techn $15: 211-214$

Gao Z (2005) Determination of soil heat flux in a Tibetan short-grass prairie. Boundary-Layer Meteorol 114:165-178

Gao Z, Fan X, Bian L (2003) An analytical solution to onedimensional thermal conduction-convection in soil. Soil Sci 168:99-107

Gao Z, Chae N, Kim J, Hong J, Choi T, Lee H (2004) Modeling of surface energy partitioning, surface temperature and soil wetness in the Tibetan prairie using the Simple Biosphere Model 2 (SiB2). J Geophys Res 109:D06102, DOI 10.1029/2003J D004089

Garratt JR (1992) The atmospheric boundary layer. Cambridge University Press, Cambridge

Heusinkvld BG, Jacobs AFG, Holtslag AAM, and Berkowicz SM (2004) Surface energy balance closure in an arid region: role of soil heat flux. Agric For Meteorol 122:21-31 
Kanemasu ET, Verma SB, Smith EA, Fritschen LY, Weseky M, Fild RT, Kustas WP, Weaver H, Steawart YB, Geney R, Panin GN, Moncrieff JB (1992) Surface flux measurements in FIFE: An overview. J Geophys Res 97:18547-18555

Kelliher FM, Köstner BMM, Hollinger DY, Byers JN, Hunt JE, McSeveny TM, Meserth R, Weir PL, Schulze ED (1992) Evaporation xylem sap flow, and tree transpiration in a New Zealand broad-leaved forest. Agric For Meteorol 62:53-73

Kustas WP, Prueger JH, Humes KS, Starks PJ (1999) Estimation of surface heat fluxes at field scale using surface layer versus mixed layer atmospheric variables with radiometric temperature observations. J Appl Meteorol 38:224-238

Kustas WP, Prueger JH, Hatfield JL, Ramalingam K, Hipps LE (2000) Variability in soil heat flux from a mesquite dune site. Agric For Meteorol 103:249-264

Lee X (1998) On micrometeorological observations of surface-air exchange over tall vegetation. Agr For Meteor 39:39-49

Lee X, Black TA (1993) Atmospheric turbulence within and above a Douglas-fir stand. Part U: Eddy fluxes of sensible heat and water vapor. Boundary-Layer Meteorol 64:369-389

Liu H, Foken T (2001) A modified Bowen ratio method to determine sensible and latent heat fluxes. Meteorol Z 10:71-80

Ma Y, Su Z, Koike T, Yao T, Ishikawa H, Ueno K, Menenti M (2003) On measuring and remote sensing surface energy partitioning over the Tibetan Plateau-from GAME/Tibet to CAMP/Tibet. Phy Chem Earth 28:63-74

Oncley SP, Foken T, Vogt R, Bernhofer C, Kohsiek W, Liu H, Pitacco A, Grantz D, Ribeiro L, Weidinger T (2002) The energy balance experiment BBEX-2000. 15th Conference on Boundary Layer and
Turbulence. American Meteorological Society, The Netherlands, 2002

Panin GN, Tetzlaff G, Raabe A, Schönfeld HJ, Nasonov AE (1996) Inhomogeneity of the land surface and the parameterization of surface fluxes-a discussion. Wiss Mitt Inst Meteorol Univ Leipzig Inst Troposphärenforsch Leipzig 4:204-215

Stull RB (1988) An introduction to boundary layer meteorology. Kluwer, Dordrecht

Tanaka K, Ishikawa H, Hayashi T, Tamagawa I (2001) Surface energy budget at Amdo on the Tibetan Plateau using GAME/Tibet IOP98 data. J Meteorol Soc Jpn 79(1B):505-517

Tanaka K, Tamagawa I, Ishikawa H, Ma Y, Hu Z (2003) Surface energy budget and closure of the eastern Tibetan Plateau during the GAME/Tibet IOP 1998. J Hydrol 283:169-283

Twine TE, Kustas WP, Norman JM, Cook DR, Houser PR, Meyers TP, Prueger JH, Starks PJ, Wesely ML (2000) Correcting eddycovariance flux underestimates over a grassland. Agric For Meteorol 103:279-300

Unland HE, Houser PH, Shuttleworth WJ, Yang ZL (1996) Surface flux measurement and modeling at a semi-arid Sonoran Desert site. Agric For Meteorol 82:119-153

Van Wijk WR (eds) (1963) Physics of Plant Environment. North Holland, Amsterdam

Wilson K, Goldstein A, Falge E, Aubinet M, Baldocchi D, Berbigier P, Bernhofer C, Ceulemans R, Dolman H, Field C, Grelle A, Ibrom A, Law BE, Kowalski A, Meyers T, Moncrieff J, Monson R, Oechel W, Tenhunen J, Valentini R, Verma S (2002) Energy balance closure at FLUXNET sites. Agric For Meteorol 113:223-243 